\title{
Acknowledgment of Authors
}

The Editors and Staff wish to sincerely thank the following authors who wrote articles for Journal of Oncology Practice in 2011.

Abernethy, Amy P.

Abesada-Terk, Guillermo Jr

Abraham, Jame

Adams, John Lloyd

Ahluwalia , Sangeeta C.

Ahmed , Tamim

Albuquerque, Kevin V.

Aleman, Carlos

Alfano, Catherine M.

Anderson, Roger

Anick, Peter

Antonio, Anna Liza M.

Armstrong, Joanne

Au, Diana

Back, Anthony L.

Baer, Allison R.

Bainbridge, Daryl

Baldwin, Laura-Mae

Barber, Beth

Barkley, Ronald R.

Barr, Thoma s R.

Basch, Ethan

Beardmore, Chris David

Beasley, Mary Beth

Beckjord, Ellen B.

Behle, Caroline M.

Bellardita, Lara

Bepler, Gerold

Berghout, Koralee

Bettello, Phyllis

Beveridge, Roy

Blanke, Charles D.

Bodnari, Andreea

Bodurtha, Joann N.

Bosserman, Linda D.

Boyd-Rogers, Stephanie

Brandt, Patricia S.

Brockstein, Bruce E.

Broder, Michael

Brown, Richard H.

Bruix, Jordi

Bugarini, Roberto

Buia, Stephanie

Bull, Janet

Burke, Lola

Burzawa, Jennifer

Busby, Leslie T.

Campion, Francis X.

Carey, Elise C.
Carro, George W.

Cartwright, Julia

Cartwright, Thomas H.

Catalano, Robert

Cavanaugh, Kristen M.

Chang, David

Chao, Calvin

Chen, Allen R.

Chen, Beth

Cheville, Andrea L.

Chien, Rebecca

Christensen, Scott D.

Chuang, Fukai L.

Chuk, Karen

Chung, Eugene

Chung, Kuo-Piao

Clark, Steven

Clayton, Matthew P.

Cohen, Gary

Connor, Maureen

Corman, Michelle

Cox, John V.

Cox, Lisa Sanderson

Currow, David C.

Daouphars, Mikael

Day, Fiona L.

Debono, David J.

deVere-White, Ralph W.

de Wilton Marsh, Robert

Devine, Susan

Dilts, David M.

Donegani, Simona

Doreau, Christian

Downey, William

Duncan, Ian

Duncan, Paul R.

Duncombe, Deborah

Dunn, Ernest

Earle, Craig C.

Eck, Carol

Egerton, Nancy J.

Elder, Elisabeth Edstrom

Elkin, Elena B.

Espirito, Janet L.

Etzioni, Ruth B.

Evans, Sarah E.

Evans, William

Faig, Douglas

Ferrusi, Ilia L.
Fetter, Alison

Fitch, Kathryn

Flamm, Carole

Fletcher, Michelle

Flum, David R.

Flynn, Thomas

Friel, Kay

Frisk, Linda

Fulp, William J.

Gadkari, Mrinalini

Gallagher, Thomas J.

Gany, Francesca

Ganz, Patricia A.

Gao, Sue

Garey, Jody

Gay, Ashley

Gesme, Dean H.

Ginsburg, Aimee

Giuse, Dario A.

Glare, Paul

Goldstein, Adam

Good, Marge

Goodin, Susan

Grabner, Michael

Graff, John J.

Granovsky, Svetlana

Gray, Catherine

Gray, Jhanelle E.

Gregory, Christopher

Griffith, Niesha

Guidi, Teri U.

Gunn, Nathan

Haas, Barbara K.

Haas, Jennifer $S$.

Hahn, Erin E.

Hajovsky, Jane

Hall, Ronald G. II

Hamaguchi, Tetsuya

Hanna, Tracie

Harper, Abigail J.

Harshberger, Cara A.

Hassett, Michael J.

Hausman, Jennifer

Hawley, Sarah T.

Hay, Sally Baron

Heifetz, Laurence J.

Hensing, Thomas

Herbeck, Eileen B.

Herbert, Carole
Hesketh, Paul J.

Hesse, Bradford W.

Hochheiser, Louis

Hohneker, John

Holcombe, Dawn

Holder, Gwen

Hong, Pengyu

Hornberger, John

Hortobagyi, Gabriel N.

Horwitz, Jill R.

Hoverman, J. Russell

Huang, Marilyn

Hui, Wendy C.

Husain, Nadia

Jacobsen, Paul B.

Jagsi, Reshma

Jain, Rahul

Johnson, Lenora E.

Johnsrud, Michael

Kadlubek, Pamela J.

Kahn, Katherine L.

Kamal, Arif H.

Kaminer, Lynne

Kan, Jeffrey $\mathrm{T}$.

Karaca-Mandic, Pinar

Kato, Ken

Katz, Steven J.

Kaur, Judith S.

Kavalieratos, Dio

Keeler, Elizabeth

Keohane, Carol

Khandekar, Janardan

Khandelwal, Nikhil

Killam, H. William

Kim, Benjamin

Kim, George P.

Kimmel, Gary

Kincheloe, Michael J.

Ko, Clifford Y.

Kohlwes, Jeff

Kolodziej, Michael

Kopp, Terrance J.

Kowaleski, Brenda

Krebs, Katie

Kris, Mark G.

Kruger, Scott

Kulin, Nathalie A.

Kunitoh, Hideo

Kuo, Raymond N.C. 
Lai, Mei-Shu

Laronga, Christine

Larson, Leanne R.

Lawton, Jessica M.

LeBlanc, Thomas W.

Lee, Ji-Hyun

Legant, Patricia

Lehmann, Christoph U.

Leighl, Natasha B.

Leng, Jennifer

Leonard, Colleen E. Ryan

Levine, Richard $\mathrm{M}$.

Levy, Mia A.

Liang, Su-Ying

Lin, James T.

Link, Emma

Lippard, Giles

Liu, Heshan

Lobach, Iryna

Lopes-Serrao, Monique D.

Lorenz, Karl A.

Lu, Karen

Lyman, Gary H.

Lynch, Patrick

Lyss, Alan P.

Malafa, Mokenge

Malin, Jennifer L.

Manasanch, Elisabet E.

Mangram, Alicia

Markham, Merry-Jennifer

Marlowe, Ursula

Marshall, Deborah A.

Martelli-Reid, Lorraine

Martz, Elaine

Massarweh, Nader N.

Massett, Holly A.

Matsumura, Yasuhiro

McCullough, Jeffrey S.

McDade, Theodore P.

McGuinness, Margaret

McKinney, Jeannine

McNatt, Wendy J.

McNish, Thelma

Meier, Klaus

Merriman, Jan

Meyer, Fremonta L.

Meyers, Fredrick J.

Milbourne, Andrea

Miller, Alexis A.

Miller, Robert S.

Milton, Daniel T.
Miovic, Michael K.

Miyake, Todd

Moore, Katrina

Morgan, Glen

Morris, Arden

Moynihan, Timothy J.

Mullins, C. Daniel

Muro, Kei

Nagashima, Kengo

Nakajima, Takako Eguchi

Neubauer, Marcus A.

Neuss, Michael N.

Newcomer, Lee N.

Nierodzick, Mary Lynn

Nutt, Stephanie

Obel, Jennifer

Okon, Ted

Oliveira, Kristin

Onukwugha, Eberechukwu

Oratz, Ruth

O’Reilly, Eileen M.

Ory, Caron

Ostroff, Jamie

Owens, William L.

Padberg, Rose Mary

Paivanas, Thomas A.

Palmer, Eric Scott

Park, Elyse Richelle

Park, James O.

Parreco, Linda K.

Partridge, Ann H.

Patel, Rinku A.

Patt, Debra A.

Pegus, Cheryl

Peteet, John R.

Peterson, Susan K.

Phillips, Kathryn A.

Phippard, Lynda

Pond, Greg

Pottow, John A.E.

Presant, Cary A.

Prestrud, Ann Alexis

Proctor, Julian W.

Pyenson, Bruce

Quesenbery, Whitney

Quillin, John M.

Rainville, Rebecca

Rajurkar, Swapnil P.

Ramirez, Julia

Rechis, Ruth

Remick, Scot C.
Richmond, Ellen S.

Rienzo, Marie E.

Rischin, Danny

Roeske, John C.

Rose, Danielle E.

Rosenquist, Joel

Rubinstein, Elan

Rudge, Nancy K.

Ruegg, Suzanne R.

Ruetten, Victoria L.

Saltz, Leonard

Samsa, Gregory P.

Sarna, Linda

Schapira, Lidia

Schenken, Larry L.

Scher, Kevin S.

Schnipper, Lowell E.

Schnoll, Robert A.

Schreiber, Fred

Schutt, David

Schwartz, Rowena

Schwartzberg, Lee

Sellers, Thomas P.

Semple, Deborah S.

Seow, Hsien

Shah, Nilay D.

Shah, Sachin R.

Shah-Mehta, Shilpa

Sharp, Philip V.

Shelby, Rebecca A.

Sheth, Sheetal

Shibata, David

Shimada, Yasuhiro

Shiovitz, Stacey

Shirao, Kuniaki

Shulman, Lawrence

Siddiqui, Mustaqeem A.

Siegel, Erin M.

Siminoff, Laura A.

Singh, Prabhjyot

Sitarik, Mark

Sklarin, Nancy T.

Skrzypczak, Stanley

Sloan, Jeff A.

Smith, Dee Anna

Smith, Jillian K.

Smith, Thomas J.

Smuck, Bobbi

Somerfield, Mark R.

Song, Xue

Spath, Wayne E.
Spatuzzi, Andrea L.

Spencer, Justin

Stabler, Stacy

Stone, Susan

Streeter, Sonya Blesser

Suh, Sang-Yeon

Sun, Charlotte

Sussman, Jonathan

Swetz, Keith M.

Symons, Rebecca Gaston

Tamés, Maria José

Tanai, Chiharu

Tanvetyanon, Tawee

Taylor, Donald H. Jr

Temin, Sarah

Terkola, Robert

Thursky, Karin

Tisnado, Diana M.

Tomkins, Julia E.

Toscano, Michele

Towle, Elaine L.

Trosman, Julia R.

Tseng, Jennifer F.

Ussery, Sarah M. Gressett

Vadnais, Barbara

Valdagni, Riccardo

Van Allen, Eliezer M.

Van Bebber, Stephanie L.

Van De Wege, Christine

Van Houten, Holly

Van Poznak, Catherine H.

Virani, Shamsuddin

Von Roenn, Jamie H.

Walling, Anne M.

Ward, Jeffery C.

Warner, Jeremy L.

Weingart, Saul N.

Willen, Michael A.

Wiseman, Marian

Wright, Debbie

Xue, Nianwen

Yamada, Yasuhide

Yamamoto, Seiichiro

Yeung, Raymond S. W.

Yu, Peter Paul

Yzquierdo, Rebecca

Zelenetz, Andrew D.

Zhao, Zhongyun

Zon, Robin 